Sportwiss 2013 - 43:83-84

DOI 10.1007/s12662-013-0302-z

Online publiziert: 15. Juni 2013

๑) Springer-Verlag Berlin Heidelberg 2013

\section{Ralf Brand ${ }^{1}$. Claudia Voelcker-Rehage ${ }^{2}$}

${ }^{1}$ Department für Sport- und Gesundheitswissenschaften, Universität Potsdam, Potsdam, Deutschland

${ }^{2}$ Jacobs Center für Lebenslanges Lernen, Jacobs University Bremen, Bremen, Deutschland

\title{
Zeit der Veränderung
}

Liebe Leserinnen und Leser,

das vergangene Jahr brachte für die Sportwissenschaft einige Veränderungen mit sich, von denen wir hoffen, dass diese auch Ihrer Einschätzung nach zu einer positiven Wegveränderung der Zeitschrift geführt haben.

Eine in unseren Augen wichtige, aktuelle Veränderung ist, dass - wie Sie vielleicht schon durch den entsprechenden Hinweis auf dem Heftumschlag bemerkt haben - die Sportwissenschaft mit ihren Beiträgen nun in der internationalen Literaturdatenbank Scopus gelistet wird. Besonderer Dank gilt dabei unserem Verlagspartner Springer, der uns dies durch sein Wirken ermöglicht hat! Zweifellos verbessert dies die internationale Visibilität der in der Sportwissenschaft publizierten Beiträge erheblich. Für unsere Autoren erwarten wir durch diesen Schritt einen noch größeren Anreiz, ihre Manuskripte bei uns einzureichen. Auch vervielfachen sich so die Chancen, mit der eigenen Arbeit von Kolleginnen und Kollegen im In- und Ausland wahrgenommen bzw. zitiert zu werden und somit zur Erweiterung des sportwissenschaftlichen Forschungsstandes beitragen zu können.

Zusätzlichen Anreiz, sich mit eigenen wissenschaftlichen Beiträgen an der Weiterentwicklung sowohl der Zeitschrift als auch des (inter)nationalen Erkenntnisstandes zu beteiligen, wollen wir durch Veränderungen der in der Sportwissenschaft publizierten Beitragsformate schaffen. Autoren haben zukünftig die Wahl zwischen Hauptbeitrag, Kurzbeitrag und Übersicht sowie drei Varianten sonstiger Beiträge.

Mit einem als Hauptbeitrag (Main Article) publizierten Artikel verfolgt die Sportwissenschaft das Ziel, einen Originalbeitrag zur Erweiterung des aktuellen internationalen Forschungsstands zu leisten. Im Mittelpunkt steht ein von den Autoren selbst erbrachter Forschungsertrag, der theoretisch elaboriert und, der jeweiligen Fachkonvention entsprechend, ausführlich dargestellt ist. Wir ermuntern Autorinnen und Autoren ausdrücklich zu erwägen, ob sie diese Art Beitrag in englischer Sprache (und damit in international rezipierbarem Format) verfassen wollen.

Mit einem als Kurzbeitrag (Brief Report) eingereichten und publizierten Manuskript verfolgt die Sportwissenschaft das Ziel, kurz, prägnant und aktuell Ergebnisse zu kommunizieren. Diese Beitragsart eignet sich besonders für die Darstellung empirischer Untersuchungen, steht grundsätzlich jedoch auch andersartigen Forschungsbeiträgen (sofern es sich um Originalia handelt) offen. Eine angemessene theoretische Verankerung ist Voraussetzung für die Darstellung. Im Vergleich zum Hauptbeitrag sollte diese jedoch knapper gefasst werden. Auch hier ermuntern wir die Autorinnen und Autoren ausdrücklich zu erwägen, ob sie ihren Beitrag in englischer Sprache verfassen wollen.

Mit einem als Übersicht (Review) publizierten Artikel verfolgt die Sportwissenschaft das Ziel, durch eine systematische Zusammenfassung und Auswertung bereits publizierter Arbeiten, Leserinnen und Lesern die Bewertung des aktuellen Forschungsstandes zu definierten Gegenstandsbereichen oder Wissensgebieten zu ermöglichen. Wir freuen uns über das Engagement von Autorinnen und Autoren, solche Arbeiten in englischer Sprache zu verfassen.

Ausdrücklich sei jedoch betont, dass Beiträge, die in deutscher Sprache verfasst wurden, nach wie vor willkommen bleiben!
Darüber hinaus sind wir auch weiterhin an der Publikation von Essays, Kommentaren, Diskussionsbeiträgen, Leserbriefen und Buchbesprechungen interessiert. Im Mittelpunkt von Beiträgen der Rubriken Essay, Kommentar und Diskussion sollte die Darstellung einer begründeten Position von Autorinnen oder Autoren stehen, die zu einem definierten und relevanten Gegenstandsbereich oder Wissensgebiet Stellung nehmen. Leserbriefe dienen der Sportwissenschaft dazu, in sehr kurzer Form und aktuell zu in früheren Ausgaben der Zeitschrift publizierten Inhalten Stellung zu nehmen. Buchbesprechungen sollten sich auf Werke beziehen, die Themen behandeln, welche möglichst nicht nur für Kolleginnen und Kollegen einer einzelnen Fachdisziplin relevant, sondern für Sportwissenschaftlerinnen und Sportwissenschaftler verschiedener Disziplinen gleichermaßen interessant sind. Für Buchbesprechungen empfehlen wir, im Vorfeld Kontakt mit den Herausgebern der Zeitschrift aufzunehmen, um abzuschätzen, welche Publikationschancen dem zu besprechenden Werk eingeräumt werden können.

Nähere Informationen, etwa zu den jeweilig möglichen und gewünschten Seitenumfängen, finden Sie unter den Autorenhinweisen auf der bekannten Internetseite der Sportwissenschaft (www. editorialmanager.com/spwi), die zur Einreichung jeglicher Beiträge eingerichtet ist.

Ein letzter, nach „innen“, also auf die Entwicklung unserer Fachdisziplin Sportwissenschaft in Deutschland gerichteter Punkt, bezieht sich auf den im vorliegenden Heft prominent platzierten Diskussionsbeitrag von Martin Lames (TU München), Mark Pfeifer (Johannes GutenbergUniversität Mainz), Andreas Hohmann 


\section{Editorial}

(Universität Bayreuth) und Andrea Horn (Bundesinstitut für Sportwissenschaft, Bonn), „Erklärung zur Lage der universitären Sportwissenschaft“. Wir bedanken uns bei diesen Autoren sehr herzlich für das der Zeitschrift entgegengebrachte Vertrauen, den Beitrag und die sich hoffentlich daraus ergebende Debatte begleiten zu dürfen. Wir freuen uns auf die Einreichung von Anschlussbeiträgen durch Kolleginnen und Kollegen, die mit uns am Führen einer ganz sicher fruchtbaren Diskussion in den nächsten Heften interessiert sind! Darüber hinaus haben wir auch im vorliegenden Heft wieder Beiträge zu unterschiedlichen Facetten sportwissenschaftlicher Forschung für Sie zusammenstellen können.

Viel Freude und vor allem Gewinn beim Lesen, wünschen Ihnen

Ralf Brand und Claudia Voelcker-Rehage (Geschäftsführende Herausgeber der Sportwissenschaft)

\section{Korrespondenzadresse}

Prof. Dr. R. Brand

Department für Sport-

und Gesundheitswissenschaften

Universität Potsdam

Am Neuen Palais 10, 14469 Potsdam

ralf.brand@uni-potsdam.de 